Portland State University

PDXScholar

Physics Faculty Publications and Presentations

Physics

9-1-1979

\title{
Brownian Motion in a Flowing Fluid
}

John D. Ramshaw

Portland State University, jdramshaw@yahoo.com

Follow this and additional works at: https://pdxscholar.library.pdx.edu/phy_fac

Part of the Physics Commons

Let us know how access to this document benefits you.

\section{Citation Details}

J.D. Ramshaw, "Brownian motion in a flowing fluid," Phys. Fluids 22, 1595 (1979)

This Article is brought to you for free and open access. It has been accepted for inclusion in Physics Faculty Publications and Presentations by an authorized administrator of PDXScholar. Please contact us if we can make this document more accessible: pdxscholar@pdx.edu. 


\title{
Brownian motion in a flowing fluid
}

\author{
John D. Ramshaw \\ Theoretical Division. University of California, Los Alamos Scientific Laboratory, Los Alamos, New \\ Mexico 87545 \\ (Received 30 October 1978; final manuscript received 7 May 1979)
}

A phenomenological theory is developed for Brownian motion in a flowing incompressible fluid. The Brownian particles are regarded as an ideal gas subject to a position- and time-dependent force field that represents interactions with the host fluid. Ths approach immediately leads to deterministic partial differential equations of motion for the Brownian particles. These equations are then examined in the limit of large friction, in which they imply an expression for the diffusional mass flux of Brownian particles. This expression is a sum of terms representing concentration, forced, thermal, and pressure diffusion. Comparisons are made with earlier work, and with the corresponding expression for the molecular diffusion flux of one component in a binary ideal-gas mixture. The Brownian and molecular diffusion fluxes are found to be identical in form, with the Brownian-particle volume fraction corresponding fo the molecular mole fraction.

\section{INTRODUCTION}

This article describes a simple phenomenological theory for Brownian motion in a flowing incompressible fluid. The principal result of the theory is an expression for the diffusional mass flux of the Brownian particles.

The foundations of the theory are laid in Sec. II. It is argued that the Brownian particles may be expected to behave like an ideal gas (whose local temperature is that of the host fluid) subject to a position- and timedependent force field. The force field represents the dynamical interaction of the Brownian particles with the fluid, as well as any external forces that may be present. This approach leads at once to deterministic partial differential equations governing the mean motion of the Brownian particle cloud. It has the advantage that the underlying random processes need not be considered explicitly. Assumptions about the stochastic character oi these processes are therefore unnecessary.

The equations of motion for the Brownian particles are, of course, coupled to those for the host fluid, and both sets of equations must be taken together to obtain a closed system. In Sec. III we examine the behavior of this system in the limit of large frictional force be tween the particles and the fluid. In this limit the mean particle and fluid velocities become very nearly equal, and the motion of the particles relative to the fluid becomes diffusional in character. An explicit expression for the diffusional mass flux of the Brownian particles is obtained. This expression is a sum of terms representing the effects of concentration, forced, thermal, and pressure diffusion. When this result is combined with the continuity equation for the particles, one obtains a generalized Smoluchowski equation in which all four types of diffusion are simultaneously accounted for.

The literature on Brownian motion has become voluminous. Most of it, of course, is concerned with Brownian motion in a uniform quiescent fluid. Although this is not the case of interest here, a brief discussion of some of this work may help to put the present approach into perspective.
Einstein ${ }^{1}$ obtained the diffusion coefficient for the Brownian particles by an ingenious application of equilibrium principles. This approach did not involve stochastic considerations or assumptions. It was largely superseded by stochastic theories based on the Langevin equation. ${ }^{2}$ These theories required assumptions of a stochastic character, but provided a much more detailed physical description. In essence, the basic stochastic assumption was that Brownian motion is a Gaussian Markov process. Based on this assumption, a mature mathematical theory evolved. ${ }^{3}$ Eventually, however, it became apparent that this theory was physically unsatisfactory because it did not take into account the (transient) virtual mass and BassetBoussinesq forces..$^{4-13}$ The inclusion of these forces, which, in general, are not negligible, causes the process to become non-Markovian. The stochastic theory is then beset by new complications, ${ }^{14-20}$ not all of which have yet been fully explored.

The complications of non-Markovian behavior can be avoided by augmenting the description so that the random process again becomes Markovian, ${ }^{13,21-23}$ but the augmented description itself is more complicated than the original one. Alternatively, these complications can be avoided by returning to the Einstein approach, or some appropriate modification thereof, and accepting a less detailed physical description of the process. ${ }^{24,25}$ This, in essence, is the spirit of the present work. Although our development is quite different from Einstein's, both are based on the recognition that limited but nontrivial information about the Brownian motion can be obtained by simple deterministic arguments, without entering into stochastic considerations.

The high-friction or diffusional limit, which we discuss in Sec. III, has also been examined recently by Wilemski ${ }^{26}$ and Titulaer. ${ }^{27}$ Both of these investigations were conducted within the framework of the classical stochastic theory, ${ }^{3}$ in which the virtual mass and Basset-Boussinesq forces are neglected. In contrast, we include these forces at the outset, and show explicitly that they become negligible in the high-friction limit. 
In addition to the phenomenological theories, there have been several more fundamental investigations of Brownian motion using the techniques of modern nonequilibrium statistical mechanics. ${ }^{28-35}$ This work will not be discussed here.

Several authors have dealt specifically with various aspects of Brownian motion in nonuniform fluids. ${ }^{36-41}$ This work, and its relation to the present results, is discussed in Sec. IV.

In Sec. $V$ we compare our result for the diffusion flux of Brownian particles with the well-known expression for the molecular diffusion flux of one component in a binary ideal-gas mixture. ${ }^{42}$ The Brownian and molecular diffusion fluxes are found to be identical in form, with the Brownian-particle volume fraction corresponding to the molecular mole fraction. This agreement is somewhat surprising, since the two cases are quite different physically.

\section{EQUATIONS OF MOTION}

Consider a large number of identical spherical Brownian particles, each of mass $m$, immersed in a flowing incompressible host fluid. The particle number density at position $\mathbf{r}$ and time $t$ is denoted by $n(\mathbf{r}, t)$. The partial mass density of the particles is then $\rho_{p}(\mathbf{r}, t)=m n(\mathbf{r}, l)$. The mean velocity field of the particles is denoted by $\mathrm{u}_{\mathrm{p}}(\mathrm{r}, t)$.

The Brownian particles interact with the host fluid and with external fields, but are assumed to be sufficiently dilute that they do not interact with each other. Let $T(\mathbf{r}, t)$ be the temperature field of the fluid, let $\mathbf{F}(\mathbf{r}, l)$ be the mean force per unit mass on the particles at $(\mathbf{r}, l)$ due to the fluid, and let $\mathrm{G}_{p}(\mathbf{r}, t)$ be the total external force per unit mass (e.g., gravitational, electromagnetic) on the particles at $(\mathbf{r}, t)$. The fundamental assumption of our theory is that the entire interaction of the fluid with the particles can be expressed in terms of the fields $T(\mathrm{r}, t)$ and $\mathbf{F}(\mathbf{r}, t)$. Under this assumption, the particles cannot distinguish between the actual physical situation of interest and the hypothetical situation in which the local temperature is constrained to be $T(\mathbf{r}, t)$, the fluid is absent, and the external force per unit mass is $\mathrm{F}(\mathrm{r}, t)+\mathrm{G}_{p}(\mathrm{r}, t)$. In this latter situation the Brownian particles behave as an ideal gas, since they do not interact with each other.

We may therefore regard the Brownian particles as an ideal gas whose local temperature is $T(\mathrm{r}, t)$, subject to an external force field $\mathbf{F}(\mathbf{r}, t)+\mathrm{G}_{p}(\mathbf{r}, t)$. The pressure in this hypothetical ideal gas is

$$
q(\mathbf{r}, l)=n(\mathbf{r}, t) k T(\mathbf{r}, t)=(k / m) \rho_{p}(\mathbf{r}, t) T(\mathbf{r}, t),
$$

where $k$ is Boltzmann's constant. (According to van't Hoff's law, $q$ is also the local osmotic pressure of the Brownian particles in the particle-fluid system.) The motion of the hypothetical ideal gas is governed by the continuity and momentum equations of fluid dynamics,

$$
\frac{\partial \rho_{p}}{\partial t}+\nabla \cdot\left(\rho_{p} \mathbf{u}_{p}\right)=0
$$

$$
\rho_{p}\left(\frac{\partial \mathbf{u}_{p}}{\partial l}+\mathbf{u}_{p} \cdot \nabla \mathbf{u}_{\phi}\right)=-\nabla q+\rho_{p} \mathbf{F}+\rho_{p} G_{p}
$$

where viscous stresses have been neglected, for simplicity. The mean motion of the Brownian particle cloud is determined by Eqs. (2) and (3), with $q$ defined by Eq. (1). These equations form a closed system if $T(\mathbf{r}, l)$ and $\mathbf{F}(\mathbf{r}, l)$ are regarded as known.

To proceed further it is necessary to specify the form of the fluid-particle interaction force field $\mathbf{F}(\mathbf{r}, t)$, which must include all forces that are important in the physical problem of interest. We shall take $\mathbf{F}(\mathbf{r}, t)$ to be

$$
\begin{aligned}
\mathbf{F}(\mathbf{r}, l)= & \beta\left(\mathbf{u}_{f}-\mathbf{u}_{p}\right)-\left(\mathbf{1} / \rho_{p}^{0}\right) \nabla p-(\eta / m) \nabla \ln T \\
& +\mathbf{F}_{V}(\mathbf{r}, l)+\mathbf{F}_{B}(\mathbf{r}, l) .
\end{aligned}
$$

where $\beta$ is the Stokes friction coefficient, $u_{f}(r, t)$ is the mean fluid velocity field, $\rho_{p}^{0}$ is the mass density of the pure particulate material, $p(\mathbf{r}, t)$ is the true pressure field in the particle-fluid system, $\eta$ is the thermophoretic force coefficient, ${ }^{13,14} \mathbf{F}_{V}(\mathbf{r}, t)$ represents the virtual mass force, ${ }^{45.46}$ and $\mathbf{F}_{B}(\mathbf{r}, l)$ represents the Basset-Boussinesq force..$^{45,46}$ In terms of the particle radius $R$ and the fluid viscosity $\mu$, we have

$$
\begin{aligned}
\beta=6 \pi \mu R / m, \\
\rho_{p}^{0}=3 m /\left(4 \pi R^{3}\right) . \\
\mathbf{F}_{V}(\mathbf{r}, l)=\left(\rho_{f}^{0} / 2 \rho_{p}^{0}\right)\left[\dot{\mathbf{u}}_{f}(l)-\dot{\mathbf{u}}_{p}(t)\right], \\
\mathbf{F}_{B}(r, t)=\left[(9 / 2 \pi)\left(\rho_{f}^{0} / \rho_{p}^{0}\right) \beta\right]^{1 / 2} \\
\times \int_{-\infty}^{t} d l^{\prime}\left(l-l^{\prime}\right)^{-1 / 2}\left[\dot{\mathbf{u}}_{f}\left(l^{\prime}\right)-\dot{\mathbf{u}}_{p}\left(t^{\prime}\right)\right] .
\end{aligned}
$$

where $\rho_{f}^{0}$ is the mass density of the pure fluid. Here, $\mathbf{u}_{f}$ and $\dot{\mathrm{u}}_{\mathrm{p}}$ denote the total time derivatives of $\mathbf{u}_{f}$ and $\mathrm{u}_{p}$ taken along the particle trajectory passing through $(\mathrm{r}, t)$. Equation (4) is not the most general possible form for $\mathbf{F}(\mathbf{r}, t)$ (for example, it neglects diffusiophore$\operatorname{sis}^{44}$ and stressphoresis ${ }^{40,41}$ forces), but it is sufficiently general for our purposes. In any case, the inclusion of additional force terms in Eq. (4) would necessitate only trivial modifications to the subsequent development.

The second term in the right member of Eq. (4) represents the force on a particle due to the ambient pressure gradient. This term is the source of the buoyant force on a particle in a stationary fluid in a gravitational field, and failure to include it may lead to serious errors.

\section{THE LIMIT OF LARGE FRICTION}

In order to examine the limit of large friction, it is necessary to consider the overall momentum equation for the particle-fluid system. Provided that $u_{p}$ and $u_{f}$ are not very different, this equation takes the familiar form

$$
\rho\left(\frac{\partial \mathbf{v}}{\partial t}+\mathbf{v} \cdot \nabla \mathbf{v}\right)=-\nabla p+\rho_{p} \mathrm{G}_{p}+\rho_{f} \mathrm{G}_{f}
$$

where $\rho_{f}(\mathbf{r}, t)$ is the partial mass density of the fluid, $\mathrm{G}_{f}(\mathbf{r}, t)$ is the total external force per unit mass on the fluid at $(\mathbf{r}, t), \rho(\mathbf{r}, t)$ is the total mass density of the par- 
ticle-fluid system,

$$
\rho(\mathbf{r}, t)=\rho_{p}(\mathbf{r}, t)+\rho_{f}(\mathbf{r}, t),
$$

$\mathbf{v}(\mathbf{r}, t)$ is the mass-averaged velocity field,

$$
\mathbf{v}(\mathbf{r}, t)=\left(\rho_{p} \mathbf{u}_{p}+\rho_{f} \mathbf{u}_{f}\right) / \rho,
$$

and viscous stresses have again been neglected, for simplicity.

The limit of large friction is accomplished by letting $\beta \rightarrow \infty$. We shall not consider the question of how large $\beta$ must be for this limit to be achieved in practice, nor are we concerned with the transient initial-condition effects that cause the limit to be approached nonuniformly in the neighborhood of the initial time. We simply rely on the intuitive expectation, justified $a$ posteriori, that a diffusional description will result in the limit $\beta \rightarrow \infty$. Notice that $\beta \rightarrow \infty$ as $R-0$.

In the limit $\beta \rightarrow \infty$, Eq. (4) implies that $u_{p}-u_{f}$ must tend to zero at least as rapidly as $\beta^{-1}$; otherwise, the term $\beta\left(u_{p}-u_{f}\right)$ would not remain finite. Equations (7) and (8) then immediately imply that

$$
\begin{aligned}
& \mathbf{F}_{V}(\mathbf{r}, t)=\mathcal{O}\left(\beta^{-1}\right), \quad(\beta \rightarrow \infty) \\
& \mathbf{F}_{B}(\mathbf{r}, t)=\mathcal{O}\left(\beta^{-1 / 2}\right) .
\end{aligned}
$$

The virtual mass and Basset-Boussinesq forces therefore vanish in the limit of large friction. This explains why the conventional stochastic theory, ${ }^{3}$ in which these forces are neglected, nevertheless yields the correct diffusion coefficient. (An alternative and complementary explanation of this circumstance was recently suggested by Batchelor. ${ }^{25}$ )

\section{Using Eq. (11), we find that}

$$
u_{p}-v=\left(\rho_{f} / \rho\right)\left(u_{p}-u_{f}\right),
$$

which implies that $u_{p}-v$ also tends to zero at least as rapidly as $\beta^{-1}$ in the limit $\beta \rightarrow \infty$. In this limit, therefore, $\mathbf{u}_{p}$ may be replaced by $\mathbf{v}$ in the left member of Eq. (3). This replacement, together with Eq. (9), yields

$$
\rho F+\rho_{f}\left(G_{p}-G_{f}\right)=\left(\rho / \rho_{p}\right) \nabla q-\nabla p .
$$

Combining Eq. (14) with Eqs. (1) and (4), and using the fact that $\mathbf{F}_{V}$ and $\mathbf{F}_{B}$ now vanish, we obtain

$$
\mathrm{J}_{p}=-D \nabla \rho_{p}-D_{T} \nabla \ln T+D_{p} \nabla p+\left(\rho_{p} \rho_{f} / \rho \beta\right)\left(\mathrm{G}_{p}-\mathrm{G}_{f}\right),
$$

where $J_{p}$ is the diffusional mass flux of Brownian particles relative to the fluid,

$$
J_{p}=\rho_{p}\left(\mathbf{u}_{p}-\mathbf{u}_{f}\right) \text {, }
$$

$D$ is the diffusion coefficient found by Einstein,

$$
D=k T / m \beta \text {, }
$$

$D_{T}$ is the thermal diffusion coefficient,

$$
D_{T}=\rho_{p} D(1+\eta / k T),
$$

and $D_{p}$ is the coefficient of pressure diffusion,

$$
D_{D}=\left(\rho_{p} / \rho-\alpha_{p}\right) / \beta=\left(1-\alpha_{p}\right)\left(1-\rho_{f}^{0} / \rho_{p}^{0}\right)\left(\rho_{\rho} / \rho \beta\right) ;
$$

here, $\alpha_{p}$ is the local particle volume fraction, defined by

$$
\alpha_{p}(\mathbf{r}, t)=\rho_{p}(\mathbf{r}, t) / \rho_{p}^{0} .
$$

Equation (15) is our basic result for the diffusional mass flux of the Brownian particles. The net flux is the sum of terms representing concentration, thermal, pressure, and forced diffusion. The pressure diffusion term vanishes when $\rho_{p}^{0}=\rho$; i.e., when $\rho_{p}^{0}=\rho_{f}^{0}$. Thus, there is no pressure diffusion when the pure particulate material has the same density as the pure fluid. The forced diffusion term vanishes when $\mathrm{G}_{p}=\mathrm{G}_{f}$; i.e., when the external force per unit mass on the particles is the same as that on the fluid. Thus, there is no forced diffusion when the only external force is that of gravity. Sedimentation and centrifugation of Brownian particles are therefore driven by pressure diffusion rather than forced diffusion, just as in the molecular case. ${ }^{47}$

For theoretical purposes, it is often preferable to deal with a diffusion flux $\mathrm{J}_{p}^{*}$ defined relative to $\mathrm{v}$ rather than $\mathrm{u}_{f}$,

$$
\mathbf{J}_{p}^{*}=\rho_{p}\left(\mathbf{u}_{p}-\mathbf{v}\right) \text {. }
$$

It follows from Eq. (13) that $J_{p}$ and $J_{p}^{*}$ are simply related by

$$
\mathbf{J}_{p}^{*}=\left(\rho_{\boldsymbol{f}} / \rho\right) \mathbf{J}_{p} .
$$

It is also sometimes preferable to regard $\nabla\left(\rho_{p} / \rho\right)$, rather than $\nabla \rho_{\phi}$, as the driving force for concentration diffusion. Since the fluid is incompressible, these two gradients are simply proportional to one another,

$$
\nabla \rho_{p}=\left(1-\alpha_{p}\right)\left(\rho^{2} / \rho_{f}\right) \nabla\left(\rho_{p} / \rho\right) .
$$

Combining Eqs. (15), (19), (22), and (23), we find that $\mathrm{J}_{\mathrm{p}}^{*}$ can be expressed in the form

$$
\begin{aligned}
\mathrm{J}_{p}^{*}= & -\rho D^{*} \nabla\left(\rho_{p} / \rho\right)-D_{T}^{*} \nabla \ln T \\
& +\frac{\rho_{f} m D^{*}}{\left(1-\alpha_{p}\right) \rho k T}\left[\left(\frac{\rho_{p}}{\rho}-\alpha_{p}\right) \nabla p+\left(\frac{\rho_{f} \rho_{p}}{\rho}\right)\left(\mathrm{G}_{p}-\mathrm{G}_{f}\right)\right],
\end{aligned}
$$

where

$$
\begin{aligned}
& D^{*}=\left(1-\alpha_{p}\right) D=\left(1-\alpha_{p}\right) k T / m \beta, \\
& D_{T}^{*}=\left(\frac{\rho_{f}}{\rho}\right) D_{T}=\frac{\rho_{f} \rho_{p}}{\left(1-\alpha_{p}\right) \rho} D^{*}\left(1+\frac{\eta}{k T}\right) .
\end{aligned}
$$

The particle continuity equation, Eq. (2), can be rewritten in terms of either $\mathbf{J}_{\rho}$ or $\mathbf{J}_{p}^{*}$,

$$
\begin{aligned}
& \frac{\partial \rho_{p}}{\partial t}+\nabla \cdot\left(\rho_{p} \mathbf{u}_{f}\right)=-\nabla \cdot \mathbf{J}_{p}, \\
& \frac{\partial \rho_{p}}{\partial t}+\nabla \cdot\left(\rho_{p} \mathbf{v}\right)=-\nabla \cdot \mathbf{J}_{p}^{*} .
\end{aligned}
$$

Equations (27) and (28) are convective diffusion equations, either of which may be regarded as the generalization of the Smoluchowski equation ${ }^{3}$ to the case of Brownian motion in a flowing fluid.

In the limit of large friction, the fundamental equations of motion for the particle-fluid system may be taken to be Eqs. (28) and (9), with the diffusion flux $\mathbf{J}_{p}^{*}$ determined by Eqs. (22) and (15). To obtain a closed system, one must add to them the equation for overall mass conservation,

$$
\frac{\partial \rho}{\partial t}+\nabla \cdot(\rho \mathbf{v})=0
$$


and an energy equation to determine the temperature $T(\mathbf{r}, t) .{ }^{47}$ The energy equation and Eqs. (9), (28), and (29) then constitute four equations in the four unknown functions $T, \mathrm{v}, \rho_{p}$, and $p$. [Since $\rho_{p}^{0}$ and $\rho_{f}^{0}$ are assumed known, $\rho_{p}$ determines $\rho_{f}$ and $\rho$ via Eqs. (10) and (20) and the relation $\rho_{f}=\left(1-\alpha_{p}\right) \rho_{f}^{0}$.]

\section{COMPARISONS WITH EARLIER WORK}

In its entirety, Eq. (15) for $\mathrm{J}_{p}$ appears to be new. However, several authors have considered various as pects of Brownian motion in nonuniform fluids, and it is of interest to compare the individual terms in $\mathrm{Eq}$. (15) with the corresponding earlier results.

Nicolis ${ }^{36}$ restricts his attention to concentration and thermal diffusion, and he evidently considers the host fluid to be stationary. Using the methods of nonequilibrium statistical mechanics, he recovers the usual Einstein relation for $D$, and obtains a formal expression for $D_{T}$. [A factor of $(3 k T)^{-1}$ appears to be missing from the right side of Nicolis' Eq. (1), and $\mu_{b}$ should be replaced by $n_{b}$ in his Eq. (8).] To lowest order in the mass ratio $\gamma$, his result for $D_{T}$ is of the form of our Eq. (18) with

$$
\eta=k T \Delta_{1}
$$

where $\Delta_{1}$ is the integral of a certain time-correlation function. Since $\eta$ is simply a phenomenological coefficient in the present theory, no further comparison is possible. Nicolis' expression for $\Delta_{1}$, together with Eq. (30), constitutes a fluctuation-dissipation theorem $\mathrm{m}^{15}$ for the thermophoretic force coefficient $\eta$.

Brownian motion in a nonuniform fluid has also been considered by Éfros. ${ }^{37}$ He does not allow for the presence of physical external force fields, and hence obtains no information about forced diffusion. Within this restriction, he considers the problem from two points of view. He first considers the case in which the pressure field is uniform and the host fluid may be either a gas or a liquid. The result of the analysis is that $D$ is given by the Einstein relation, Eq. (17), and $D_{T}$ is given by our Eq. (18) with $\eta=0$. Thus, Efros has, in effect, neglected the thermophoretic force.

The second case considered by Éfros is that in which $\nabla p$ may be nonzero, but the host fluid is an ideal gas. The analysis of this case leads to the same expressions for $D$ and $D_{T}$ as those found in the first case, and also predicts that

$$
D_{p}=\left(m / m_{0}\right) \rho_{p} D / p,
$$

where $m_{0}$ is the mass of a single gas molecule. Since the Brownian particles are assumed to be very dilute, $p$ is essentially equal to $\left(\rho / m_{0}\right) k T$. Equation (31) may therefore be rewritten as

$$
D_{p}=\rho_{p} / \rho \beta,
$$

where use has been made of Eq. (17). Comparison with Eq. (19) shows that Éfros' result for $D_{p}$ lacks the term $-\alpha_{p} / \beta$. This term arises from the direct pressure force on the Brownian particles. [It is easy to verify that if the second term in the right member of Eq. (4) had been omitted, we would have obtained Eq. (32) instead of Eq. (19).] The second equality in Eq. (19) shows that the term $-\alpha_{p} / \beta$ is negligible only when the host fluid is a low-density gas, with $\rho_{f}^{0} \ll \rho_{p}^{0}$, and the Brownian particles are sufficiently dilute so that $\alpha_{p}$ $\ll 1$. This was indeed the case considered by Éfros. However, the use of Eq. (32) when $\alpha_{\rho}$ is comparable to $\rho_{p} / \rho$ would lead to serious errors, a limitation that was not revealed by Éfros' analysis.

The fact that Eq. (19), rather than Eq. (32), is the correct general form for $D_{p}$, can be confirmed by considering a spatially uniform distribution of neutrally buoyant particles $\left(\rho_{p}^{0}=\rho_{f}^{0}\right)$ in a stationary isothermal fluid in a gravitational field $G$. In this situation the concentration, thermal, and forced diffusion terms all vanish. Since the particles are neutrally buoyant, it is physically clear that the total diffusion flux $J_{p}$ will also vanish. Therefore, the pressure diffusion term $D_{p} \nabla p$ must be zero. But, hydrostatic equilibrium requires that $\nabla p=\rho \mathrm{G} \neq 0$. Thus, $D_{p}$ must be zero for neutrally buoyant particles, a condition that is fulfilled by Eq. (19), but not by Eq. (32).

Zubarev and Bashkirov ${ }^{38,39}$ derive a Fokker-Planck equation for Brownian motion in a flowing fluid, and also evaluate the diffusion flux of the particles relative to the fluid. For the latter purpose they specialize (although it seems unnecessary) to the case of a uniform pressure field. Consequently, they do not obtain the pressure diffusion flux. They also omit external force fields, and hence do not obtain the forced diffusion flux. Zubarev and Bashkirov find that $D$ is given by the Einstein relation, Eq. (17), and that $D_{T}$ is of the form of our Eq. (18) with

$$
\eta=k T \Delta_{1}-(4 \pi / 3) R^{3} p,
$$

where $\Delta_{1}$ is the same correlation-function integral as that appearing in the theory of Nicolis. ${ }^{36}$

Both Nicolis and Zubarev and Bashkirov obtain expressions for the Brownian particle diffusion flux which are consistent with the phenomenological theory of the present article. However, they disagree on the proper microscopic expression for the net thermophoretic force coefficient $\eta$. For reasons explained in the Appendix, we believe that the Nicolis result, Eq. (30), is correct while the result of Zubarev and Bashkirov, Eq. (33), is in error.

$\mathrm{MazO}^{40}$ developed a theory of Brownian motion in a nonuniform fluid that extends the work of Nicolis and Zubarev and Bashkirov. Mazo's principal result is a Fokker-Planck equation which generalizes that of Zubarev and Bashkirov. He does not examine the diffusional limit, and hence does not obtain expressions for $J_{p}$ or the diffusion coefficients.

Brownian motion in a nonuniform gas has been studied by Slinn and Shen. ${ }^{41}$ The analysis is restricted to the case in which the Knudsen number based on particle radius is much larger than unity. The gas molecules are assumed to experience specular reflection at the surface of the Brownian particles. Under these restrictions, Slinn and Shen obtain a Fokker-Planck 
equation for the Brownian-particle distribution function. They also examine the diffusional limit, in which they obtain a convective diffusion equation for the number density of Brownian particles. This equation [obtained by combining Eqs. (77) and (63) of Slinn and Shen] can be written in the form of Eq. (27), thereby enabling $\mathbf{J}_{p}$ to be identified. If the viscous stresses are neglected, the resulting expression for $\mathrm{J}_{p}$ becomes

$$
\mathbf{J}_{p}=-D \nabla \rho_{p}-D_{T} \nabla \ln T+\left(\rho_{p} / \beta\right) \mathrm{G}_{p} .
$$

Here, $D$ and $D_{T}$ are still given by Eqs. (17) and (18), but $\beta$ is now given by the Epstein formula [Eq. (28) of Slinn and Shen] instead of Eq. (5), and $\eta$ is given by

$$
\eta=(m \beta \lambda T / 5 p)
$$

where $\lambda$ is the thermal conductivity of the host gas. The replacement of the Stokes $\beta$ by the Epstein $\beta$ is, of course, to be expected here, and $\mathrm{Eq} .(35)$ is indeed the correct expression for the thermophoretic force coefficient in the case of present interest. ${ }^{44}$ Comparison of Eqs. (34) and (15) then shows that the theory of Slinn and Shen and the present theory are in agreement with regard to concentration and thermal diffusion, but not with regard to pressure and forced diffusion.

The nature of this disagreement is clarified by examining the effect of neglecting the mass averaged acceleration $(\partial v / \partial t+v \cdot \nabla v)$ in the present theory. Equation (9) then reduces to

$$
\nabla p=\rho_{p} \mathrm{G}_{b}+\rho_{f} \mathrm{G}_{f},
$$

which combines with Eqs. (15) and (19) to yield

$$
\mathrm{J}_{p}=-D \nabla \rho_{p}-D_{T} \nabla \ln T+\left(\rho_{p} / \beta\right) \mathrm{G}_{p}-\left(\alpha_{p} / \beta\right) \nabla p .
$$

We have already seen that the last term in Eq. (37) arises from the direct pressure force on the Brownian particles, and that this term is negligible when $\rho_{f}^{0} \ll \rho_{p}^{0}$ and $\alpha_{p} \ll 1$. We may therefore omit this term in the rarefied-gas regime of interest to Slinn and Shen, provided that the Brownian particles are sufficiently dilute. Equation (37) then becomes identical to the expression of Slinn and Shen for $J_{\Delta}$, Eq. (34). We therefore conclude that Slinn and Shen have effectively neglected the local mass-averaged acceleration of the particle-fluid mixture. This approximation is entirely unrelated to the diffusional limit, as is clear from the development of the preceding section.

This concludes our comparisons with earlier work. In summary, we note that (a) there is general agreement on the concentration diffusion coefficient; (b) there is some disagreement about the thermophoretic part of the thermal diffusion coefficient; and (c) none of the work discussed ${ }^{36-41}$ provides a general treatment of pressure and forced diffusion. However, the correct form of the pressure diffusion flux is not unknown in other contexts. It has previously been obtained in connection with two-phase flow, ${ }^{48}$ but its relevance to Brownian motion has apparently not been appreciated.

\section{COMPARISON WITH MOLECULAR DIFFUSION}

It is of interest to compare the diffusional mass flux of Brownian particles with the corresponding molecular diffusion flux in a binary ideal-gas mixture. Let the two molecular species be A and B. According to the kinetic theory of gases, ${ }^{42}$ the diffusive mass flux of the molecular species A relative to the mass-averaged velocity may be cast into the form

$$
\begin{aligned}
\mathbf{J}_{A}^{*}= & -\rho D_{A B} \nabla\left(\rho_{A} / \rho\right)-D_{A}^{T} \nabla \ln T \\
& +\frac{\rho_{B} m_{A} D_{A B}}{\left(1-x_{A}\right) \rho k T}\left[\left(\frac{\rho_{A}}{\rho}-x_{A}\right) \nabla p+\frac{\rho_{A} \rho_{B}}{\rho}\left(G_{A}-G_{B}\right)\right],
\end{aligned}
$$

where $D_{A B}$ is the binary diffusivity of the pair AB, $D_{A}^{T}$ is the thermal diffusion coefficient, $x_{A}$ is the mole fraction of species $A, m_{A}$ is the mass of a single $A$ molecule, $\rho_{A}$ and $\rho_{B}$ are the partial mass densities of species $A$ and $B$, respectively, $G_{A}$ and $G_{B}$ are the external forces per unit mass acting on species $A$ and $B$, respectively, and $\rho=\rho_{A}+\rho_{B}$. Comparison with Eq. (24) shows that the molecular and Brownian diffusion fluxes are of identical form, with the mole fraction of species $A$ in the molecular case corresponding to the volume fraction of the particles in the case of Brownian motion. This circumstance is really quite remarkable, since the two cases are very different physically.

\section{CONCLUDING REMARKS}

A phenomenological theory of Brownian motion in a flowing fluid has been developed. In the limit of large friction, the theory leads to a new expression for the diffusional mass flux of the Brownian particles. This expression is a sum of terms representing concentration, forced, pressure, and thermal diffusion.

Our development has been based on the use of Eq. (4) to represent the mean force per unit mass $F(r, t)$ of the fluid on the particles. This form for $\mathbf{F}(\mathbf{r}, t)$ was felt to include most of the important physical effects in the problem. It is clear that the development would proceed along the same outline if a more general form for $\mathbf{F}(\mathbf{r}, t)$ were adopted, in which case a correspondingly more general form for the diffusion flux would result.

The assumption that the Brownian particles are in local thermodynamic equilibrium with the fluid is an essential ingredient of our theory. This assumption is tenable only when the characteristic time for attaining this equilibrium (which is of order $\left.\beta^{-1}\right)^{3}$ is much shorter than that over which the fluid temperature changes along a particle trajectory. The equation of motion for the particles, Eq. (3), is therefore valid only when

$$
(\beta T)^{-1}\left|\partial T / \partial t+\mathbf{u}_{p} \cdot \nabla T\right| \ll 1 .
$$

In general, this condition will differ from that for the validity of the diffusional description. However, the diffusional description is unconditionally valid in the limit $\beta-\infty$, since Eq. (39) is identically satisfied in that limit.

It is hoped that the present theory will find application in biophysics, colloid science, aerosol science, and related areas. 


\section{ACKNOWLEDGMENTS}

I am grateful to J. K. Dukowicz and L. G. Margolin for many helpful discussions, to R. M. Mazo and R. W. Zwanzig for directing my attention to some of the relevant earlier work, and to J. T. Hynes for clarifying some of that work.

This work was performed in part under the auspices of the United States Department of Energy.

\section{APPENDIX}

Here, we examine the theory of Zubarev and Bashkirov $^{38,39}$ in greater detail. A comparison of their Fokker-Planck equation with the conventional FokkerPlanck equation for Brownian motion in an external force field ${ }^{3}$ shows that the mean force per unit mass of the fluid on the particles in their theory is given by

$$
\mathbf{F}(\mathbf{r}, t)=\beta\left(\mathbf{u}_{f}-\mathbf{u}_{p}\right)+\left\langle\mathscr{F}_{1}\right\rangle_{l}^{\prime} / m-\left(k T \Delta_{1} / m\right) \nabla \ln T,
$$

where $\left\langle\mathscr{F}_{1}\right\rangle_{1}^{\prime}$ is the "local equilibrium" force of the fluid on a representative particle, which Zubarev and Bashkirov approximate by

$$
\left\langle\mathscr{F}_{1}\right\rangle_{l}^{\prime}=-(4 \pi / 3) R^{3} k T \nabla(p / k T) .
$$

Equation (A2) is Eq. (13) of Ref. 39, rewritten in our notation with the sign error corrected. If we combine Eqs. (A1) and (A2) and compare the result with Eq. (4), we again find that the net thermophoretic force coefficient, according to Zubarev and Bashkirov, is given by Eq. (33). (We also see that Zubarev and Bashkirov have omitted the virtual mass and Basset-Boussinesq forces, whose relevance to Brownian motion was not then understood. This, of course, is how they are able to obtain a Markovian Fokker-Planck equation.)

From a macroscopic point of view, Eq. (A2) is a curious relation, for it involves $\nabla(p / T)$ instead of $\nabla p$. We therefore try to reconstruct the argument that led to it. Zubarev and Bashkirov appear to have arrived at Eq. (A2) by considering the special case of a fixed particle surrounded by a nonuniform ideal gas in local thermodynamic equilibrium. The local number density of gas molecules in the presence of the particle is then given approximately by

$$
n_{\boldsymbol{g}}(\mathbf{r})=n_{g}^{0}(\mathbf{r}) \exp [-V(\mathbf{r}) / k T(\mathbf{r})],
$$

where $V(\mathbf{r})$ is the interaction energy of the fixed particle with a gas molecule located at the point $r$, and $n_{g}^{\circ}(\mathbf{r})$ is the number density of the gas in the absence of the particle. The ambient pressure field of the gas is simply given by

$$
p(\mathbf{r})=n_{g}^{0}(\mathbf{r}) k T(\mathbf{r}) .
$$

In terms of $n_{g}(\mathbf{r})$, the force $\left\langle\mathscr{F}_{1}\right\rangle_{l}^{\prime}$ is given by

$$
\left\langle\mathscr{F}_{1}\right\rangle_{l}^{\prime}=\int d \mathbf{r} n_{g}(\mathbf{r}) \nabla V(\mathbf{r}) .
$$

Combining Eqs. (A5) and (A3), and making use of the identity

$$
\begin{aligned}
& \exp (-V / k T) \nabla V \\
& \quad=k T \nabla[1-\exp (-V / k T)]+V \exp (-V / k T) \nabla \ln T,
\end{aligned}
$$

we obtain

$$
\begin{aligned}
\left\langle\mathscr{F}_{1}\right\rangle_{l}^{\prime}= & -\int d \mathbf{r}[1-\exp (-V / k T)] \nabla p(\mathbf{r}) \\
& +\int d \mathbf{r} V \exp (-V / k T) n_{\boldsymbol{p}}^{0}(\mathbf{r}) \nabla \ln T(\mathbf{r})
\end{aligned}
$$

where use has been made of Eq. (A4), and an integration by parts has been performed.

Since the particles are much larger than the gas molecules, a negligible error is incurred by regarding $V(\mathbf{r})$ as a hard sphere potential, which is infinite inside the particle and zero elsewhere. The last term in Eq. (A7) then vanishes identically, and we obtain

$$
\left\langle\mathscr{F}_{1}\right\rangle_{l}^{\prime}=-(4 \pi / 3) R^{3} \nabla p
$$

where we have made use of the fact that $\nabla p$ is essentially uniform over distances of order $R$. Equation (A8) states that $\left\langle\mathscr{F}_{1}\right\rangle_{i}^{\prime}$ is simply the ambient pressure force that one would have written down macroscopically, and which is represented by the second term in the right member of Eq. (4). We therefore conclude that the expression of Zubarev and Bashkirov for $\left\langle\mathscr{F}_{1}\right\rangle_{l}^{\prime}, \mathrm{Eq}$. (A2), is in error, and should be replaced by Eq. (A8). If Eq. (A8) is combined with $\mathrm{Eq}$. (A1), a comparison of the result with $\mathrm{Eq}$. (4) shows that $\eta$ is correctly given by the Nicolis result, Eq. (30), rather than the result of $\mathrm{Zub}$ arev and Bashkirov, Eq. (33).

${ }^{1}$ A. Einstein, Ann. Phys. (Leipz.) 17, 549 (1905); reprinted in Investigations on the Theory of the Brownian Movement (Dover, New York, 19.56).

${ }^{2}$ P. Langevin, C. R. Acad. Sci. (Paris) 146, 530 (1908).

${ }^{3}$ S. Chandrasekhar, Rev. Mod. Phys. 15, 1 (1943); reprinted in Selected Papers on Noise and Stochastic Processes, edited by N. Wax (Dover, New York, 1954).

${ }^{4}$ R. Zwanzig and M. Bixon, Phys. Rev. A 2, 2005 (1970).

${ }^{5}$ R. Zwanzig and M. Bixon, J. Fluid Mech. 69, 21 (1975).

${ }^{6}$ A. Widom, Phys. Rev. A 3, 1394 (1971).

${ }^{7}$ T. S. Chow and J. J. Hermans, J. Chem. Phys. 56, 3150 (1972).

${ }^{8}$ T. S. Chow and J. J. Hermans, J. Chem. Phys. 57, 1799 (1972).

${ }^{9}$ T. S. Chow and J. J. Hermans, J. Chem. Phys. 58, 825 (1973).

${ }^{10}$ T. S. Chow and J. J. Hermans, Physica 65, 156 (1973).

${ }^{11}$ J. T. Hynes, J. Chem. Phys. 57, 5612 (1972).

${ }^{12}$ M. Nelkin, Phys. Fluids 15, 1685 (1972).

${ }^{13} \mathrm{~J}$. W. Dufty, Phys. Fluids 17, 328 (1974).

${ }^{14} \mathrm{R}$. Kubo, in Many-Body Theory, edited by R. Kubo (Syokabo/ Benjamin, Tokyo/New York, 1966), p. 1.

${ }^{15}$ R. Kubo, Rept. Progr. Phys. 29, 255 (1966).

${ }^{16} \mathrm{R}$. Kubo, in Transport Phenomena, edited by G. Kirezenow and J. Marro (Springer-Verlag, New York, 1974), p. 74.

${ }^{17}$ S. A. Adelman, J. Chem. Phys. 64, 124 (1976).

${ }^{18}$ S. A. Adelman and B. J. Garrison, Mol. Phys. 33, 1671 (1977).

${ }^{19}$ R. F. Fox, J. Stat. Phys. 16, 259 (1977).

${ }^{20}$ R. F. Fox, J. Math. Phys. 18, 2331 (1977).

${ }^{21}$ E. H. Hauge and A. Martin-Löf, J. Stat. Phys. 7, 259 (1973).

${ }^{22}$ D. Bedeaux and P. Mazur, Physica 76, 247 (1974).

${ }^{23}$ E. J. Hinch, J. Fluid Mech. 72, 499 (1975).

${ }^{24} \mathrm{G}$. K. Batchelor, J. Fluid Mech. 74, 1 (1976).

${ }^{25} \mathrm{G}$. K. Batchelor, in Theoretical and Applied Mechanics, edited by W. T. Koiter (North-Holland, Amsterdam, 1976), p. 33 ${ }^{26} \mathrm{G}$. Wilemski, J. Stat. Phys. 14, 153 (1976).

${ }^{27}$ U. M. Titulaer, Physica A 91, 321 (1978). 
${ }^{28}$ J. L. Lebowitz and E. Rubin, Phys, Rev. 131, 2381 (1963). ${ }^{29}$ P. Resibois and H. T. Davis, Physica 30, 1077 (1964).

${ }^{30}$ R. I. Cukier and J. M. Deutch, Phys. Rev. 177, 240 (1969).

${ }^{31}$ P. Mazur and I. Oppenheim, Physica 50, 241 (1970).

${ }^{32} \mathrm{~J}$. Albers, J. M. Deutch, and I. Oppenheim, J. Chem. Phys. 54, 3541 (1971).

${ }^{33}$ J. T. Hynes, R. Kapral, and M. Weinberg, Physica A 80, 105 (1975).

${ }^{34}$ J. T. Hynes, R. Kapral, and M. Weinberg, Physica A 81, 485 (1975).

${ }^{35}$ J. T. Hynes, R. Kapral, and M. Weinberg, Physica A 81, 509 (1975).

${ }^{36}$ G. Nicolis, J. Chem. Phys. 43, 1110 (1965).

${ }^{37}$ A. L. Éfros, Zh. Eksp. Teor. Fiz. 50, 809 (1966) [Sov. Phys.JETP 23, 536 (1966)].

${ }^{38}$ D. N. Zubarev and A. G. Bashkirov, Phys. Lett. A 25, 202 (1967).
${ }^{39}$ D. N. Zubarev and A. G. Bashkirov, Physica 39, 334 (1968). ${ }^{40}$ R. M. Mazo, J. Stat. Phys. 1, 101 (1969).

${ }^{41}$ W. G. N. Slinn and S. F. Shen, J. Stat. Phys. 3, 291 (1971).

${ }^{42} \mathrm{~J}$. O. Hirschfelder, C. F. Curtiss, and R. B. Bird, Molecular Theory of Gases and Liquids (Wiley, New York, 1954).

${ }^{43} \mathrm{~N}$. A. Fuchs, The Mechanics of A erosols (Pergamon, Oxford, $1964)$.

${ }^{44} \mathrm{~L}$. Waldmann and K. H. Schmitt, in Aerosol Science, edited by C. N. Davies (Academic, New York, 1966), p. 137.

${ }^{45} \mathrm{C}$. M. Tchen, Doctoral Dissertation, Delft (Martinus Nijhoff, The Hague, 1947); quoted by J. O. Hinze, Turbulence (McGraw-Hill, New York, 1975), 2nd. ed., p. 46.3.

${ }^{46}$ S. Corrsin and J. Lumley, Appl. Sci. Res. A 6, 114 (1956). ${ }^{47}$ R. B. Bird, W. E. Stewart, and E. N. Lightfoot, Transport Phenomena (Wiley, New York, 1960).

${ }^{48} \mathrm{~J}$. R. Travis, F. H. Harlow, and A. A. Amsden, Nucl. Sei. Eng. 61, 1 (1976). 